\title{
Therapeutic Effect of Extracorporeal Shock Wave Therapy According to Treatment Session on Gastrocnemius Muscle Spasticity in Children With Spastic Cerebral Palsy: A Pilot Study
}

\author{
Dong-Soon Park, $\mathrm{MD}^{1}$, Dong Rak Kwon, $\mathrm{MD}^{1}$, Gi-Young Park, $\mathrm{MD}^{1}$, Michael Y. Lee, $\mathrm{MD}^{2}$
}

${ }^{1}$ Department of Rehabilitation Medicine, Catholic University of Daegu School of Medicine, Daegu, Korea; ${ }^{2}$ Department of Physical
Medicine and Rehabilitation, School of Medicine, The University of North Carolina at Chapel Hill, Chapel Hill, NC, USA

Objective To investigate the therapeutic effect of extracorporeal shockwave therapy (ESWT) according to treatment session on gastrocnemius muscle spasticity in children with spastic cerebral palsy (CP).

Methods Twelve children with spastic CP underwent 1 ESWT and 2 sham ESWT sessions for gastrocnemius (group 1) or 3 ESWT sessions (group 2) once per week for 3 weeks. Modified Ashworth Scale (MAS) score, passive range of motion (PROM) of the ankle plantar-flexor muscles with knee extension, and median red pixel intensity (RPI) of color histogram of medial gastrocnemius on real-time sonoelastography (RTS) were measured before ESWT, immediately after the first and third ESWT, and at 4 weeks after the third ESWT.

Results Mean ankle PROM was significantly increased whereas as mean ankle MAS and median gastrocnemius RPI were significantly decreased in both groups after the first ESWT. Clinical and RTS parameters before ESWT were not significantly different from those immediately after the third ESWT or at 4 weeks after the third ESWT in group 1. However, they were significantly different from those immediately after the third ESWT or at 4 weeks after the third ESWT in group 2. Mean ankle PROM, mean ankle MAS, and median gastrocnemius RPI in group 2 were significantly different from that in group 1 at 4 weeks or immediately after the third ESWT.

Conclusion The therapeutic effect of ESWT on spastic medial gastrocnemius in children with spastic CP is dependent on the number of ESWT sessions.

Keywords Spasticity, Cerebral palsy, Extracorporeal shock wave therapy, Gastrocnemius muscle, Sonoelastography

Received April 27, 2015; Accepted June 16, 2015

Corresponding author: Dong Rak Kwon

Department of Rehabilitation Medicine, Catholic University of Daegu School of Medicine, 33 Duryugongwon-ro 17-gil, Nam-gu, Daegu 42472, Korea Tel: +82-53-650-4878, Fax: +82-53-622-4687, E-mail: coolkwon@cu.ac.kr

() This is an open-access article distributed under the terms of the Creative Commons Attribution Non-Commercial License (http://creativecommons.org/ licenses/by-nc/4.0) which permits unrestricted noncommercial use, distribution, and reproduction in any medium, provided the original work is properly cited. Copyright $\odot 2015$ by Korean Academy of Rehabilitation Medicine 


\section{INTRODUCTION}

Spasticity and reduced active muscle strength are the two predominant motor problems of spastic cerebral palsy (CP) [1]. Spasticity has neural and biomechanical components. The neural component is the level of resistance to passive movement. It is a velocity-dependent phenomena. Its severity can increase up to 4 years of age and decrease thereafter up to 12 years of age [2]. The biomechanical component of spasticity is related to muscle stiffness. It is influenced by high collagen content in spastic muscle. Its severity increases with age $[3,4]$. In patients with spastic $\mathrm{CP}$, inactivity owing to muscle spasticity can lead to muscle weakness, muscle atrophy, and muscle shortening [5].

As children with spastic CP age, passive muscle stiffness may play a more important role in spasticity than reflex activity [6]. We have previously demonstrated that changes in the spasticity of the ankle plantar-flexor muscles after rehabilitation therapy and botulinum toxin type A injection are related to age [6]. Treatment of spasticity should ideally modulate muscle tone according to the mechanism of muscle stiffness. Therefore, age should be considered when establishing a treatment plan for children with spastic CP [7].

Extracorporeal shockwave therapy (ESWT) has been recently demonstrated to effective for improving spasticity in children with spastic CP [8-10]. Consequently, it has been considered as a new reversible therapeutic method for this condition. However, the mechanisms by which ESWT affects spasticity remain unclear. The reduction in spasticity after ESWT may be due to its direct action on fibrous areas by altering the rheological properties of chronically hypertonic muscles and by reducing intramuscular connective tissue stiffness [11].

Real-time sonoelastography (RTS) is a recently developed ultrasound-based technique to evaluate tissue elasticity in real time. RTS is based on the principle that tissue compression can produce strain and cause displacement, with greater strain in soft tissues than in hard tissues [12]. Our previous study has demonstrated that RTS shows differences in muscle stiffness between spastic and normal gastrocnemius (GCM) muscle in spastic CP [13]. Increased collagen content in the spastic muscle can be indirectly estimated using RTS measurements of intrinsic stiffness of the spastic muscle.
Up to date, no recommended guideline is available about the correct number of ESWT session for the treatment of chronic soft tissue lesion [14]. Cumulative effect of repeated ESWT on epidermal nerve fibers and plantar fasciitis have been previously reported $[15,16]$. To the best of our knowledge, no study has determined the changes in muscle stiffness using RTS in children with spastic CP after ESWT or the influence of the number of ESWT treatment sessions on spastic CP-related muscle stiffness. Therefore, the objective of this study was to determine whether the changes in GCM stiffness and RTS in children with spastic CP after ESWT could be affected by the number of ESWT treatment sessions.

\section{MATERIALS AND METHODS}

This study was designed as a prospective, doubleblinded, and randomized controlled trial.

\section{Patients}

Twelve children with spastic CP (8 with diplegia and 4 with hemiplegia) were recruited from the outpatient clinic of the Department of Rehabilitation Medicine at Daegu Catholic University Medical Centre. They were randomly allocated to two groups. Group 1 had 6 children (4 boys and 2 girls) at a mean age of 7.0 \pm 3.1 years. Their mean body weight was $22.5 \pm 3.3 \mathrm{~kg}$. There were 4 children with diplegia and 2 with hemiplegia with a total of 10 treated legs. Of the 6 children, 2 were at Gross Motor Function

Table 1. Demographic data of children with spastic cerebral palsy

\begin{tabular}{lcc}
\hline & Group 1 (n=6) & Group 2 (n=6) \\
\hline Age (yr) & $7.0 \pm 3.1$ & $6.8 \pm 2.3$ \\
Gender (boy:girl) & $4: 2$ & $3: 3$ \\
Body weight (kg) & $22.5 \pm 3.3$ & $21.9 \pm 2.9$ \\
$\begin{array}{l}\text { Cerebral palsy } \\
\text { (diplegic:hemiplegic) }\end{array}$ & $4: 2$ & $4: 2$ \\
GMFCS (level II:III) & $2: 4$ & $2: 4$ \\
\hline
\end{tabular}

Values are presented as mean \pm standard deviation or number.

Group 1, one true and two sham ESWT sessions; Group 2, three true ESWT sessions; GMFCS, Gross Motor Function Classification System; ESWT, extracorporeal shockwave therapy.

${ }^{*} \mathrm{p}<0.05$. 
Classification System (GMFCS) level II and 4 were at GMFCS level III. Group 2 included 6 children ( 3 boys and 3 girls) at a mean age of $6.8 \pm 2.3$ years. Their mean body weight was $21.9 \pm 2.9 \mathrm{~kg}$. There were 4 children with diplegia and 2 with hemiplegia with a total of 10 treated legs. Of the 6 children, 2 and 4 were at GMFCS level II and III, respectively (Table 1 ).

The inclusion criteria were as follows: 1) verified diagnosis of spastic $\mathrm{CP}$ by a physiatrist specializing in pediatric rehabilitation medicine, 2) independent ambulation with or without gait-assistive devices, 3) spastic gait with ankle equinus, and 4) dynamic ankle contracture. Dynamic ankle contracture was confirmed if ankle equinus was observed during ambulation. Passive dorsiflexion of the ankle could be accomplished beyond the neutral position with knee extended. The exclusion criteria were as follows: 1) older than 13 years or younger than 2 years, 2) previous botulinum toxin type A injection in the GCM or serial casting of the ankle within 6 months prior to enrolment, 3) fixed ankle contracture, and 4) surgery of the lower limbs in the past 12 months. The Institutional Review Board and Ethics Committee of Daegu Catholic University Medical Centre approved the study protocol. Since all patients were younger than 18 years, informed consent for participation was obtained from their parents or guardians.

\section{ESWT application}

An electromagnetic type Dornier AR2 machine (Dornier MedTech GmbH, Wessling, Germany) was used to apply ESWT. The pressure pulses were focused on the lowerlimb hypertonic muscles. A total of 1,500 pulses were delivered to each GCM. Pulses were mainly applied in the middle of the muscle belly under ultrasound guidance. The energy flux density was at $0.030 \mathrm{~mJ} / \mathrm{mm}^{2}$ and the repetition frequency was at $4 \mathrm{~Hz}$. For double-blinded treatment, two types of ESWT treatment were performed according to treatment session ( 1 vs. 3 sessions). Both groups had a sham or true ESWT for three consecutive weekly treatments. Group 1 received one true ESWT in session 1 and two sham ESWTs in sessions 2 and 3. Group 2 received three true ESWTs for all 3 sessions. Due to its low-energy application, ESWT is not painful. It does not require anesthesia or analgesic drugs. Adverse events were monitored throughout the treatment and after treatment. In addition, patients received outpatient reha- bilitation treatment during the treatment and after ESWT treatment, including twice weekly sessions of stretching exercises, strengthening exercises, functional electrical stimulation, and progressive gait training.

\section{Clinical measurement}

Clinical assessments were performed before, immediately after the first ESWT and the third ESWT, and at 4 weeks after the third ESWT by a physiatrist. Passive range of motion (PROM) of ankle dorsiflexion was assessed with a two-arm goniometer in supine position with the knee extended. The spasticity of ankle plantar-flexor muscles was measured as the degree of resistance to passive movement using Modified Ashworth Scale (MAS). The amount of resistance felt was rated from 0 (normative) to 5 (extreme). Gross Motor Function Measure (GMFM) was determined before treatment by a physical therapist.

\section{Real-time sonoelastography}

Ultrasonography and RTS of the medial GCM were performed simultaneously using a commercially available ultrasound system with a 5- to $13-\mathrm{MHz}$ multi-frequency linear transducer (Antares; Siemens Healthcare, Erlangen, Germany) before, immediately after the first and the third ESWT, and at 4 weeks after the third ESWT by another physiatrist with 10 years of experience in musculoskeletal ultrasound and 3 years of experience in RTS. The longitudinal plane of the medial GCM was scanned. Patients were scanned on an examination table in prone position with their feet hanging off the edge. Natural compression was applied with the transducer with normal respiration. Ultrasonography was discontinued if reflexive or voluntary contraction of lower-limb muscles was observed. The proximal tendon, musculotendinous junction, and muscle portion of the medial GCM were identified from proximal-to-distal scanning of leg on ultrasonography. Scans were performed repeatedly at a fixed point on the medial GCM between two reference points. One reference point was located at the proximal third of a longitudinal line from midway between the medial and lateral malleoli to midway between the medial and lateral epicondyles. The other reference point was located at the medial end on a transverse line perpendicular to the point on the longitudinal line. A region of interest (ROI) including the medial GCM fascicles with clearly 
demarcated linear hyperechoic strands corresponding to the fibroadipose septa (i.e., perimysium) and normal surrounding tissue was selected under ultrasound guidance.

RTS was performed to produce color-coded graphic representation of the relative stiffness of the structures within the selected ROI, with purple indicating soft, green and yellow indicating intermediate stiffness, and red indicating stiff. The degree of compression was determined by manually adjusting the transducer so that the perimysium appeared predominantly yellow, orange, or red on RTS. The color-coded RTS and ultrasound images were displayed on the left and right sides of the monitor, respectively. The RTS protocol described herein included standardized color coding for all patients.

The best representative recorded RTS image was selected based on adequate depiction of tissue structure and consistent reproduction of scanned images. The color pattern of the recorded images was quantitatively analyzed using ImageJ software (National Institutes of Health, Bethesda, MD, USA) by a different physiatrist. The color histograms generated represented the number of pixels within a fixed list of color ranges. The median red pixel intensity (RPI) within the ROI was calculated, with higher values indicating higher color intensity. RTS was performed twice. A representative image was taken during each scan to examine the intra-rater reliability of measurements.

\section{Statistical analysis}

Fisher exact test was used to compare sex differences and topographical classification of CP between groups at baseline. Intra-group changes in the PROM of ankle dorsiflexion, MAS of ankle plantar-flexors, and median RPI were analyzed with a two-factor repeated-measures analysis of variance from baseline immediately after ESWT and 4 weeks after the completion of the final session. Inter-group differences in these measured parameters were analyzed using Mann-Whitney U-test. Interclass correlation coefficients were calculated to examine the intra-rater reliability of repeated RPI measurements. The intra-class correlation coefficients (ICCs) were classified as excellent (values of 0.75 and greater), fair to good (0.44-0.75), and poor (values less than 0.44) [17]. Statistical analysis was performed using SPSS ver. 19.0 (IBM Corporation, Armonk, NY, USA). The level of significance was set at $\mathrm{p}<0.05$.

\section{RESULTS}

There was no significant difference between groups at baseline with respect to age, sex, topographical classification, GMFCS level, PROM of ankle dorsiflexion, MAS of the ankle plantar-flexor, GMFM, or median RPI of the affected medial GCM. No side effect was observed until 4 weeks after the third ESWT.

In both groups, the mean ankle MAS was decreased significantly after the first ESWT session. The mean ankle MAS in group 2 was significantly lower than that in group 1 immediately and at 4 weeks after the third ESWT ( $1.0 \pm 0.4$ vs. $2.0 \pm 0.7, \mathrm{p} \leq 0.001 ; 1.1 \pm 0.5$ vs. $2.3 \pm 0.8, \mathrm{p}=0.025$ ) (Table 2).

The mean PROM of ankle dorsiflexion in both groups was increased significantly after the first ESWT. There

Table 2. Clinical and real-time sonoelastographic parameters after ESWT

\begin{tabular}{|c|c|c|c|c|c|c|c|c|}
\hline & \multicolumn{4}{|c|}{ Group $1(n=6)$} & \multicolumn{4}{|c|}{ Group $2(n=6)$} \\
\hline & BT & AFT & ATT & 4WATT & BT & AFT & ATT & 4WATT \\
\hline MAS & $2.5 \pm 0.7$ & $1.5 \pm 0.5^{b)}$ & $2.0 \pm 0.7$ & $2.3 \pm 0.8$ & $2.3 \pm 0.4$ & $1.2 \pm 0.4^{\mathrm{b})}$ & $1.0 \pm 0.4^{\mathrm{a}, \mathrm{b})}$ & $1.1 \pm 0.5^{\mathrm{a}, \mathrm{b})}$ \\
\hline $\operatorname{PROM}\left(^{\circ}\right)$ & $5.8 \pm 2.0$ & $13.3 \pm 3.7^{b)}$ & $7.9 \pm 1.3$ & $7.3 \pm 1.8$ & $6.6 \pm 2.5$ & $13.6 \pm 3.8^{b)}$ & $22.5 \pm 2.7^{\mathrm{a}, \mathrm{b}}$ & $19.1 \pm 3.7^{\mathrm{a}, \mathrm{b}}$ \\
\hline RPI & $156.1 \pm 9.8$ & $130.7 \pm 6.1^{b)}$ & $146.6 \pm 7.4$ & $147.9 \pm 5.0$ & $158.6 \pm 8.4$ & $134.8 \pm 8.5^{\mathrm{b})}$ & $127.6 \pm 9.8^{\mathrm{a}, \mathrm{b})}$ & $136.6 \pm 7.6^{\mathrm{a}, \mathrm{b}}$ \\
\hline
\end{tabular}

Values are presented as mean \pm standard deviation.

Group 1, one true and two sham ESWT sessions; Group 2, three true ESWT sessions; ESWT, extracorporeal shockwave therapy; BT, before treatment; AFT, after the first ESWT treatment; ATT, after the third ESWT treatment; 4WAT, at 4 weeks after the third ESWT treatment; MAS, Modified Ashworth Scale; PROM, passive range of motion of ankle dorsiflexion; RPI, red pixel intensity.

${ }^{a)} \mathrm{p}<0.05$, Mann-Whitney $U$ test between group 1 and group 2 .

${ }^{b)} \mathrm{p}<0.05$, derived from repeated measures of ANOVA for assessment time. 

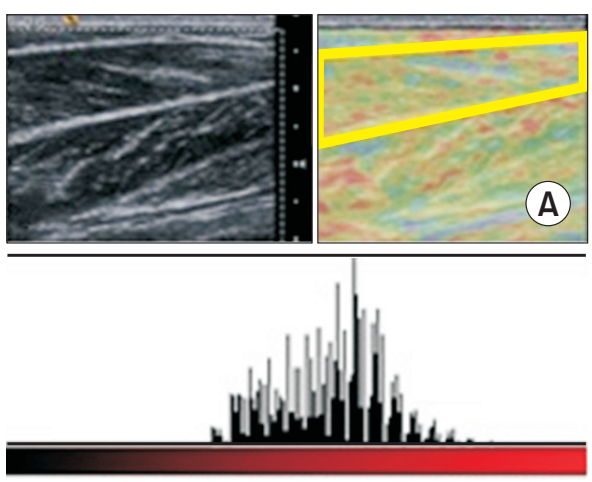

rMean: 140.46 rSD: 23.74 rMode: 151
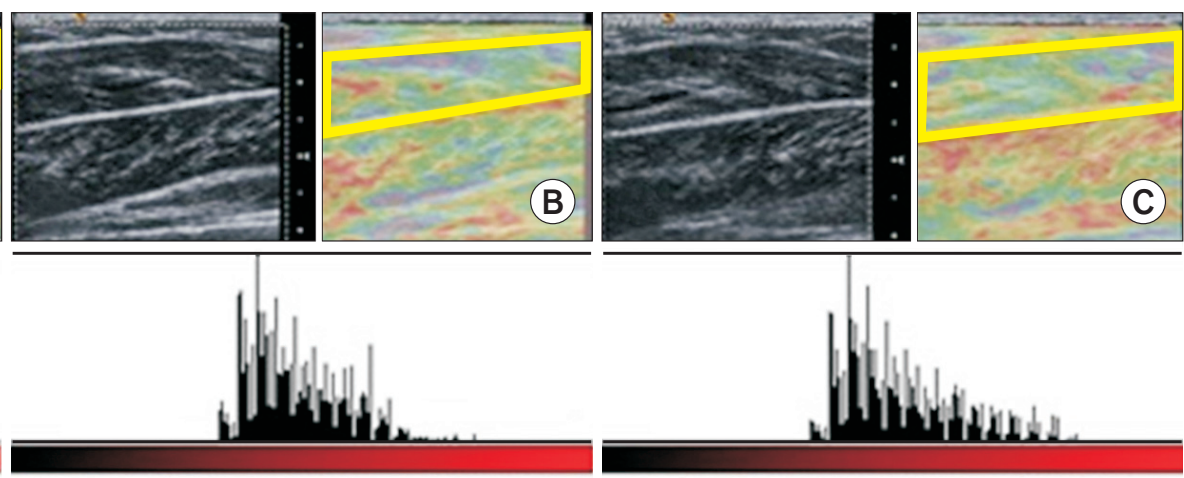

rMean: 124.21 rSD: 20.99 rMode: 107

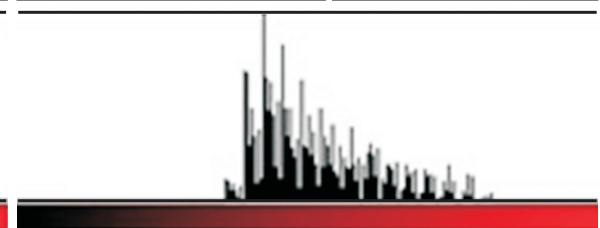

rMean: 129.42 rSD: 25.16 rMode: 107

Fig. 1. Representative longitudinal RTS image and red color histogram of medial gastrocnemius (GCM) muscle before and after three ESWT sessions. Yellow polygons and freehand selections depict the region of interest for quantitative analysis of the medial GCM stiffness on color histogram. (A) Baseline RTS image showing predominantly red color in the medial GCM muscle. The median RPI of the medial GCM was 140.4 at baseline. (B, C) Follow-up RTS images showing purple to yellow colors after ESWT. The median RPI was 124.2 at immediately after ESWT treatment (B) and 129.4 at 4 weeks after 3 weekly ESWT treatments (C). RTS, real-time sonoelastography; ESWT, extracorporeal shockwave therapy; RPI, red pixel intensity.

was no significant difference in the mean increase of PROM of ankle dorsiflexion between the two groups after the first ESWT $\left(13.3^{\circ} \pm 3.7^{\circ}\right.$ vs. $\left.13.6^{\circ} \pm 3.8^{\circ}, \mathrm{p}>0.05\right)$ (Table 2). The mean PROM of ankle dorsiflexion in group 2 was significantly higher than that in group 1 immediately and at 4 weeks after the third ESWT $\left(22.5^{\circ} \pm 2.7^{\circ}\right.$ vs. $7.9^{\circ} \pm 1.3^{\circ}$, $\mathrm{p}=0.003 ; 19.1^{\circ} \pm 3.7^{\circ}$ vs. $7.3^{\circ} \pm 1.8^{\circ}, \mathrm{p} \leq 0.001$ ) (Table 2 ).

The mean RPI of the medial GCM was decreased significantly immediately after the first ESWT in both groups. There was no significant difference in the mean RPI of the medial GCM between the two groups immediately after the first ESWT session ( $130.7 \pm 6.1$ vs. $134.8 \pm 8.5, \mathrm{p}>0.05)$ (Table 2). However, the mean RPI of the medial GCM in group 2 was significantly lower than that in group $1 \mathrm{im}$ mediately and at 4 weeks after the third ESWT (127.6 \pm 9.8 vs. $146.6 \pm 7.4, \mathrm{p} \leq 0.001 ; 136.6 \pm 7.6$ vs. $147.9 \pm 5.0, \mathrm{p}=0.015$ ) (Table 2, Fig. 1).

The ICCs of repeated RPI measurements at baseline in groups 1 and 2 were 0.889 and 0.902 , respectively. Immediately after ESWT, the ICCs of repeated RPI measurements were 0.899 and 0.895 , respectively. At 4 weeks after ESWT, the ICCs of repeated RPI measurements were 0.901 and 0.897 , respectively.

\section{DISCUSSION}

Immediately after the first ESWT, children with CP exhibited reduced ankle plantar-flexor muscle spasticity, including decreased MAS score, increased PROM, and decreased muscle stiffness on RTS. Such therapeutic effect lasted 4 weeks after the third ESWT. However, ankle plantar-flexor muscle spasticity in group 1 was increased over time, although it was not significantly different from the baseline at 4 weeks after the third sham ESWT. To our knowledge, the present study was the first to assess the influence of the number of treatment sessions of ESWT on spasticity in children with spastic CP.

Our results showed that ankle plantar-flexor muscle spasticity was decreased significantly immediately after ESWT and at 4 weeks after the third ESWT in children with spastic CP. In a previous study, the upper- and lower-limb muscles of patients with severe spastic CP (mean age, 31 years) have been treated with 3 sessions of ESWT with 2,000 pulses at $0.10 \mathrm{~mJ} / \mathrm{mm}^{2}$ [9]. It was found that the therapeutic effect was maintained until two months post-treatment [9]. However, direct comparison between the results of the previous study and the results of this study is difficult because this study had a relatively short follow-up time with younger patient age. In addition, different treatment protocol was used in the two studies.

In the present study, a single ESWT session on spastic ankle plantar-flexor muscles had an immediate therapeutic effect. However, the effect disappeared at 4 weeks after the third sham ESWT in group 1. The present results are in concordant with a study on patients with spastic post-stroke lower-limb muscle replaced by fibrosis 
(muscle echo intensity grade IV) [18]. In that study, structural changes of spastic muscle are associated with time elapsed since stroke onset. In addition, a single ESWT immediately decreased lower-limb spasticity, although that effect was not sustained [18]. On the contrary, the effect of a single ESWT with 1,500 pulses and $0.030 \mathrm{~mJ} / \mathrm{mm}^{2}$ has been reported to last 4 weeks post-treatment in children (mean age, 8 years) with spastic hemiplegic CP [8]. The difference in the duration of therapeutic effect after ESWT in different studies might be attributable to differences in spastic muscle involvement in the paretic lower limb of spastic CP. It has been reported that the majority of 8-year-old children with spastic hemiplegic CP can achieve a higher GMFM score than children in the same age group with spastic diplegic CP [19,20]. A higher total GMFM score indicates a higher rate of activity. Muscle structure and function are correlated with the rate of activity in children with CP [21]. Decreased voluntary motor unit recruitment can cause joint immobilization, which can lead to the development of muscle contracture around joints, consequently decreasing muscle mass while increasing intramuscular connective tissue content [22]. Compared to a normal muscle cell, a spastic muscle cell has a shorter resting sarcomere and a higher elastic modulus, implying dramatic remodeling of intra- and extra-cellular structural components such as titin and collagen [23]. Collagen plays an important physiological role in the development of muscle stiffness [24]. Inactivity in children with spastic CP can increase non-reducible collagen content in the spastic muscle, leading to muscle weakness, disuse muscle atrophy, and muscle shortening [5]. In the present study, 8 of 12 had spastic diplegic CP. The short-term effect of ESWT might be attributable to the high intrinsic muscle stiffness in spastic diplegic CP. Therefore, repeated ESWT treatments are required to alter intrinsic muscle stiffness with a longer duration.

The mechanism by which ESWT affects spastic muscles remains unknown. Spasticity has neural and biomechanical components. The biomechanical component is related to muscle stiffness. It is influenced by titin and collagen content in the spastic muscle. The speculated mechanism of decreased spasticity immediate after a single ESWT may be related to changed muscle physical properties (thixotropy), such as breaking the functional links between actin and myosin. Titin-actin interaction can induce reduction in intrinsic stiffness of connective tissue in chronically hypertonic muscles as a result of being mechanically shaken (vibration) $[25,26]$. In addition, it has been hypothesized that the waves in ESWT can dilate blood vessels via enzymatic promotion [10-12] and non-enzymatic nitric oxide synthesis [13], thus subsequently inducing neovascularization, increasing the blood supply to the tissue, and modulating the secretion of interleukins and the activation of growth factors.

RTS has been used to measure muscle stiffness by indirectly assessing the increased collagen content in spastic muscle. Post-treatment changes in muscle stiffness measured by RTS have been reported in children with spastic CP $[27,28]$. The present study used ultrasonography and RTS to assess the changes of muscle stiffness after ESWT and demonstrated a high intra-rater reliability of RPI measurement in the medial GCM. Our results was in concordant with a previous study [29]. The mean RPI of the medial GCM was decreased significantly immediately after ESWT in both groups. There was no significant difference between the two groups. However, the mean RPI of the medial GCM was significantly lower in children who received 3 ESWT sessions than those who received a single ESWT session at one month post-treatment. The change in intrinsic stiffness after ESWT according to RTS is similar to that of rehabilitation therapy with botulinum toxin type A injected into children with spastic CP [27].

This study has several limitations that should be addressed. First, the sample size was small and the followup period after treatment was short. Second, the interrater reliability of RPI measurement was not assessed. RTS examination is operator-dependent. It requires sufficient experience. It also has technical problems in image reproducibility. Third, the GMFM score was not assessed after ESWT. Fourth, the two groups apparently differed with respect to spastic CP involvement. Therefore, additional larger studies for children with the same spastic CP type with a longer follow-up period and GMFM assessment are required to confirm the results of this study.

Nonetheless, this pilot study revealed that the reduction of medial GCM spasticity in children with spastic CP after ESWT was dependent on the number of ESWT sessions. Our results showed that three ESWT sessions were more effective in reducing muscle stiffness than one ESWT session. Further studies with more patients and longer follow-up periods are required to confirm the clinical usefulness of ESWT and clarify the most appropriate 
treatment protocol.

\section{CONFLICT OF INTEREST}

No potential conflict of interest relevant to this article was reported.

\section{ACKNOWLEDGMENTS}

This work was supported by a grant of Research Institute of Medical Science, Catholic University of Daegu, Korea (No. 201502).

\section{REFERENCES}

1. Graham HK, Selber P. Musculoskeletal aspects of cerebral palsy. J Bone Joint Surg Br 2003;85:157-66.

2. Hagglund G, Wagner P. Development of spasticity with age in a total population of children with cerebral palsy. BMC Musculoskelet Disord 2008;9:150.

3. Johnson DC, Damiano DL, Abel MF. The evolution of gait in childhood and adolescent cerebral palsy. J Pediatr Orthop 1997;17:392-6.

4. Bell KJ, Ounpuu S, DeLuca PA, Romness MJ. Natural progression of gait in children with cerebral palsy. J Pediatr Orthop 2002;22:677-82.

5. de Paiva A, Meunier FA, Molgo J, Aoki KR, Dolly JO. Functional repair of motor endplates after botulinum neurotoxin type A poisoning: biphasic switch of synaptic activity between nerve sprouts and their parent terminals. Proc Natl Acad Sci U S A 1999;96:3200-5.

6. Pierce SR, Prosser LA, Lauer RT. Relationship between age and spasticity in children with diplegic cerebral palsy. Arch Phys Med Rehabil 2010;91:448-51.

7. Lee WY, Park GY, Kwon DR. Comparison of treatment effects between children with spastic cerebral palsy under and over five years after botulinum toxin type A injection. Ann Rehabil Med 2014;38:200-8.

8. Amelio E, Manganotti P. Effect of shock wave stimulation on hypertonic plantar flexor muscles in patients with cerebral palsy: a placebo-controlled study. J Rehabil Med 2010;42:339-43.

9. Vidal X, Morral A, Costa L, Tur M. Radial extracorporeal shock wave therapy (rESWT) in the treatment of spasticity in cerebral palsy: a randomized, placebo-controlled clinical trial. NeuroRehabilitation
2011;29:413-9.

10. Gonkova MI, Ilieva EM, Ferriero G, Chavdarov I. Effect of radial shock wave therapy on muscle spasticity in children with cerebral palsy. Int J Rehabil Res 2013;36:284-90.

11. Manganotti P, Amelio E. Long-term effect of shock wave therapy on upper limb hypertonia in patients affected by stroke. Stroke 2005;36:1967-71.

12. Park GY, Kwon DR. Application of real-time sonoelastography in musculoskeletal diseases related to physical medicine and rehabilitation. Am J Phys Med Rehabil 2011;90:875-86.

13. Kwon DR, Park GY, Lee SU, Chung I. Spastic cerebral palsy in children: dynamic sonoelastographic findings of medial gastrocnemius. Radiology 2012;263:794-801.

14. Speed CA. Extracorporeal shock-wave therapy in the management of chronic soft-tissue conditions. J Bone Joint Surg Br 2004;86:165-71.

15. Takahashi N, Ohtori S, Saisu T, Moriya H, Wada Y. Second application of low-energy shock waves has a cumulative effect on free nerve endings. Clin Orthop Relat Res 2006;443:315-9.

16. Lee SJ, Kang JH, Kim JY, Kim JH, Yoon SR, Jung KI. Dose-related effect of extracorporeal shock wave therapy for plantar fasciitis. Ann Rehabil Med 2013;37:37988.

17. Kolber MJ, Hanney WJ. The reliability and concurrent validity of shoulder mobility measurements using a digital inclinometer and goniometer: a technical report. Int J Sports Phys Ther 2012;7:306-13.

18. Santamato A, Micello MF, Panza F, Fortunato F, Logroscino G, Picelli A, et al. Extracorporeal shock wave therapy for the treatment of poststroke plantar-flexor muscles spasticity: a prospective open-label study. Top Stroke Rehabil 2014;21 Suppl 1:S17-24.

19. Harries N, Kassirer M, Amichai T, Lahat E. Changes over years in gross motor function of 3-8 year old children with cerebral palsy: using the Gross Motor Function Measure (GMFM-88). Isr Med Assoc J 2004;6:40811.

20. Beckung E, Carlsson G, Carlsdotter S, Uvebrant P. The natural history of gross motor development in children with cerebral palsy aged 1 to 15 years. Dev Med Child Neurol 2007;49:751-6.

21. Ko IH, Kim JH, Lee BH. Relationships between lower limb muscle architecture and activities and participa- 
tion of children with cerebral palsy. J Exerc Rehabil 2013;9:368-74.

22. Gracies JM. Pathophysiology of spastic paresis. I: Paresis and soft tissue changes. Muscle Nerve 2005;31:53551.

23. Friden J, Lieber RL. Spastic muscle cells are shorter and stiffer than normal cells. Muscle Nerve 2003;27:157-64.

24. Feit H, Kawai M, Mostafapour AS. Increased resistance of the collagen in avian dystrophic muscle to collagenolytic attack: evidence for increased crosslinking. Muscle Nerve 1989;12:476-85.

25. Mirea A, Onose G, Padure L, Rosulescu E. Extracorporeal shockwave therapy (ESWT) benefits in spastic children with cerebral palsy (CP). J Med Life 2014;7 Spec No. 3:127-32.
26. Shrivastava SK, Kailash. Shock wave treatment in medicine. J Biosci 2005;30:269-75.

27. Park GY, Kwon DR. Sonoelastographic evaluation of medial gastrocnemius muscles intrinsic stiffness after rehabilitation therapy with botulinum toxin A injection in spastic cerebral palsy. Arch Phys Med Rehabil 2012;93:2085-9.

28. Kwon DR, Park GY, Kwon JG. The change of intrinsic stiffness in gastrocnemius after intensive rehabilitation with botulinum toxin A injection in spastic diplegic cerebral palsy. Ann Rehabil Med 2012;36:400-3.

29. Nordez A, Gennisson JL, Casari P, Catheline S, Cornu C. Characterization of muscle belly elastic properties during passive stretching using transient elastography. J Biomech 2008;41:2305-11. 